\title{
Schizophreniform Disorder
}

\section{Background}

1. Definition:

- A psychotic disorder like schizophrenia

- Duration $<6$ months

$\circ$ Return to normal functioning expected/usual

2. General information:

- With schizophrenia, prodromal symptoms may develop over several years

- Schizophreniform (SFM) disorder has rapid progression from onset of prodromal symptoms to point where all criteria for schizophrenia (except duration and deterioration) are met (within $6 \mathrm{mo}$ )

\section{Pathophysiology}

1. Pathology of disease:

- Not completely known

- Likely related to excess dopaminergic activity

2. Incidence, prevalence:

- Incidence: $0.09 \%$

- Lifetime prevalence: $0.11 \%$

- Equally distributed between sexes

○ Peak onset between ages of 18-24 years in men and 24-35 in women

3. Risk factors:

- Family hx of schizophrenia or mood disorder

4. Morbidity / mortality:

- Approximately $2 / 3$ of patients progress to schizophrenia

- Prominent confusion during the illness

- Rapid development of symptoms

- Good previous interpersonal and goal-oriented functioning and lack of negative psychosis symptoms suggest better outcome

\section{Diagnostics}

1. History

- Chief complaint includes:

- Confusion, perplexity, thought difficulties, neutral or blunted mood, paranoia

○ HPI:

- Minimal prodrome

- Onset is acute (unlike schizophrenia)

- No precipitating stressor(s)

- Patient usually fully oriented with intact memory

2. Physical exam

○ Usually normal

3. Diagnostic testing

- Laboratory evaluation

- Electrolytes

- Thyroid profile

- Drug screen 
- B12 and serum folate

4. Diagnostic imaging

- MRI may show increased ventricle size

5. Diagnostic criteria: 3 major criteria for SFM Disorder according to DSM IV:

- At least 2 persistent positive or negative symptoms must be present, each for a significant length of time during a 1-month period:

- Delusions

- Disorganized speech which is strange, peculiar or difficult to comprehend

- Disorganized (bizarre or child-like) behavior

- Catatonic behavior

- Hallucinations

- Negative symptoms (flat affect, alogia, avolition)

- Limited duration

- Psychotic symptoms have occurred for at least 1 month but $<6$ months

○ Symptoms cannot:

- Occur as part of a mood disorder

- Occur as part of schizoaffective disorder or schizophrenia

- Be due to intoxication with drugs or alcohol

- Be an adverse reaction to medication

- Be caused by physical injury or medical illness

- Specify if patient lacks favorable prognostic features, defined as 2 or more of the following:

- Onset of prominent psychotic symptoms within 4 weeks of first noticeable change in usual behavior or functioning

- Perplexity and thought disorganization at the height of psychotic episode

- Good premorbid social and occupational functioning

- Absence of blunted or flat affect

\section{Differential Diagnosis}

1. Key DDx

- Schizophrenia

- Brief psychotic disorder

- Substance-induced psychotic disorder

- Schizoaffective Disorder

- Major depressive disorder with psychotic features

- Delusional disorder

2. Extensive DDx

- CNS:

- Infection (encephalitis), trauma, complex seizure disorder, neoplasm

○ Endocrine:

- Hyper/hypothyroid, hyper/hypoglycemic

- Drug intoxication or withdrawal:

- LSD, PCP, cocaine, benzodiazepines, amphetamines, antipsychotics, hallucinogens, alcohol

- Hypercalcemia

- B12 or thiamine deficiency 
Therapeutics (SOR:B) - similar to Tx for schizophrenia

1. Acute treatment

- Assess acuity and need for hospitalization

- Stabilize with antipsychotics as needed

2. Further management (24 hrs)

- Rule out organic syndrome or drug intoxication/withdrawal

- Assess patients ability to function

- Commonly used:

- Risperidone, paliperidone (FDA approved), aripiprazole (FDA approved), olanzapine, quetiapine and ziprasidone

- All have shown efficacy

3. Long-term care

- Discontinue antipsychotics in 3-6 mos, no maintenance medication

- Lithium, anticonvulsants, typical antipsychotics and different atypical antipsychotic if recurrent

- Individual and family psychotherapy

Follow-Up

1. Refer to specialist

- Assessment by professional with expertise in treating mood disorders recommended

2. Admit to hospital

- 1st time psychosis

- Patient poses threat to self or others

- Patient is unable to care for themselves

Prognosis (SOR:B)

1. See Diagnostic Criteria

2. Good prognostic features usually indicate affective disorders vice schizophrenia

3. According to the APA, approximately $2 / 3$ of patients diagnosed with schizophreniform disorder progress to schizophrenia.

\section{Prevention}

1. None

\section{Patient Education}

1. http://www.webmd.com/schizophrenia/guide/mental-health-schizophreniformdisorder

2. http://www.emedicinehealth.com/schizophrenia/article_em.htm

3. http://www.nlm.nih.gov/medlineplus/schizophrenia.html

\section{References}

1. American Psychiatric Association. Diagnostic and Statistical Manual of Mental Disorders, Fourth Edition, Text Revision (DSM-IV-TR). Washington, DC: American Psychiatric Association; 2000

2. Bhalla RN. Schizophreniform disorder. eMedicine, Last Updated: Aug 20, 2007. Available at: http://www.emedicine.com/MED/topic3350.htm 
3. Drake RJ, Dunn G, Tarrier N, Bentall RP, Haddock G, Lewis SW. Insight as a predictor of the outcome of first-episode nonaffective psychosis in a prospective cohort study in England. J Clin Psychiatry. Jan 2007;68(1):81-6.

4. Goldner EM, Hsu L, Waraich P, Somers JM. Prevalence and Incidence studies of schizophrenic disorders: a systemic review of the literature. Can J Psychiatry. 2002 Nov;47(9):833-43.

5. Iancu, I, P. V. Dannon, R. Ziv, and E. Lepkifker. "A follow-up study of patients with DSM-IV schizophreniform disorder." Canadian Journal of Psychiatry47, no. 1 (2000): 56-60.

Authors: Kathleen Marshall, \& Shanin Gross, MD, Penn State Hershey Medical Center, $P A$

Editor: Robert Marshall, MD, MPH, Capt MC USN, Puget Sound Family Medicine Residence, Naval Hospital, Bremerton, WA 